\title{
28 Research Square \\ Can passive leg raise predict the response to fluid resuscitation in ED?
}

Mohammed H Elwan ( $\nabla$ m.elwan@le.ac.uk)

University of Leicester https://orcid.org/0000-0002-6825-9663

\section{A Roshdy}

North Middlesex University Hospital: North Middlesex University Hospital NHS Trust

\section{EM Elsharkawy}

Alexandria Medicine: Alexandria University Faculty of Medicine

\section{SM Eltahan}

Alexandria Medicine: Alexandria University Faculty of Medicine

\section{TJ Coats}

University of Leicester

\section{Research Article}

Keywords: Preload responsiveness, Fluid therapy, Haemodynamics, Emergency, Non-invasive monitoring, bioimpedance

Posted Date: August 19th, 2022

DOI: https://doi.org/10.21203/rs.3.rs-1235100/v2

License: (c) (i) This work is licensed under a Creative Commons Attribution 4.0 International License. Read Full License 


\section{Abstract}

\section{Objective}

Passive leg raise (PLR) can be used as a reversible preload challenge to stratify patients according to preload response. We aim to evaluate the accuracy of PLR, monitored by a non-invasive cardiac output monitor in predicting to response to fluid resuscitation in emergency department (ED)

\section{Methods}

We recruited adult patients planned to receive a resuscitation fluid bolus. Patients were monitored using a thoracic electrical bioimpedance (TEB) cardiac output monitor (Niccomo, Medis, Germany). A 3-minute PLR was carried out before and after fluid infusion. Stroke volume changes $(\Delta S V)$ were calculated and a positive response was defined as $\geq 15 \%$ increase.

\section{Results}

We recruited 39 patients, of which 37 were included into the analysis. The median age was 63 (50-77) years and 19 patients were females. 17 patients (46\%) were fluid responders compared to $11(30 \%)$ with positive response to PLR1. $\triangle$ SV with PLR1 and fluid bolus showed moderate correlation $(r=0.47,95 \%$ confidence interval, $\mathrm{Cl}$ 0.17-0.69) and $62 \%$ concordance rate. For the prediction of the response to a fluid bolus the PLR test had a sensitivity of $41 \%(95 \% \mathrm{Cl} 22-64)$ and specificity of $80 \%(95 \% \mathrm{Cl} 58-92)$ with an area under the curve of $0.59(95 \% \mathrm{Cl} 0.41-0.78)$. None of the standard parameters showed a better predictive ability compared to PLR.

\section{Conclusion}

Using TEB, $\triangle S V$ with PLR showed a moderate correlation with fluid bolus, with a limited accuracy to predict fluid responsiveness. The PLR test was a better predictor of fluid responsiveness than the parameters commonly used in emergency care (such as heart rate and blood pressure). These data suggest the potential for a clinical trial in sepsis comparing TEB monitored, PLR directed fluid management with standard care.

\section{Key Messages}

\section{What is already known on this subject?}

- Both over- and under-resuscitation are associated with harm.

- Passive leg raise showed high accuracy in predicting fluid response in ICU and peri-operative settings.

- There is little evidence on the accuracy of passive leg raise in Emergency Department.

\section{What this study adds?}


- Stroke volume changes with passive leg raise are moderately correlated with fluid bolus with limited accuracy.

- Patients who had a large adverse response with fluid bolus were accurately predicted by passive leg raise.

- Passive leg raise showed better accuracy than commonly used standard parameters (e.g. heart rate and blood pressure).

\section{Introduction}

Fluid resuscitation is often the first line of treatment for circulatory failure in emergency care, often guided by resuscitation protocols to ensure timely delivery. However, one-size-fits-all protocols have not consistently demonstrated benefit over standard care. ${ }^{1}$ The varying needs of resuscitation fluids would result in patients receiving too much and others too little fluids - with the signal of benefit diluted in a heterogeneous group. This suggests that a more individualised approach to shocked patients could be the way forward for optimising resuscitation.

This challenge is most evident in sepsis resuscitation. Protocols commonly recommend a fixed volume of resuscitation fluids followed by escalation of treatment to vasopressors or inotropes. ${ }^{2}$ This protocol is recommended for all patients, however 'suspected sepsis' covers such a wide range of patients that this 'one size fits all' approach is unlikely to be correct. In Emergency Department (ED), monitoring the response to treatment relies on standard parameters (e.g. blood pressure and heart rate). These are often insensitive to changes in circulatory volume, cardiac preload or the cardiac output response to fluid resuscitation - and potentially misleading. ${ }^{3}$

Standard monitoring parameters are used as proxies for cardiac output (CO) to determine whether patients are responsive following a preload challenge (preload/fluid responsiveness). A positive response means that patients operate on the steep part of Frank-Sterling curve and could tolerate (and potentially benefit from) fluid resuscitation. ${ }^{4} \mathrm{~A}$ negative response indicates operating on the flat part of the curve and may be harmed from fluid resuscitation. While $30 \mathrm{~mL} / \mathrm{kg}$ of initial sepsis resuscitation is commonly recommended, microvascular damage can happen as early as $9 \mathrm{~mL} / \mathrm{kg}$ of fluid administered. ${ }^{5}$ There is even less room for error with patients at risk of fluid overload in ED.

With no risk of overzealous infusion, Passive leg raise (PLR), a reversible self-fluid challenge, can be used to stratify patients into responders and non-responders ${ }^{6}$ Standard parameters are often insensitive to stroke volume (SV) changes induced by PLR and direct CO monitoring is required. ${ }^{7}$ However, the advent of non-invasive cardiac output monitors rendered this approach more feasible in ED. ${ }^{8}$ PLR seems highly accurate in predicting fluid responsiveness in sedated ventilated patients in intensive care and operating room context. ${ }^{6}$ However, there is much less evidence for the accuracy of this approach in ED - where the majority of patients are awake and spontaneously breathing. ${ }^{9}$ 
In this study, we investigated the accuracy of stroke volume response to PLR, monitored by a noninvasive bioimpedance cardiac output monitor, in predicting the stroke volume response to a subsequent fluid bolus in ED.

\section{Methods}

This was a prospective observational study of a convenience sample of adult ED patients ( $\geq 18$ years old) planned to receive at least one resuscitation bolus of IV fluids by the treating team. The study was carried out at Leicester Royal Infirmary, an inner city acute hospital at Leicester, UK. The study was sponsored by the University of Leicester, UK and ethical approvals were obtained from Essex Research Ethics Committee (16/EE/0145).

Exclusion criteria were: clinical condition preventing the performance of a PLR (e.g. trauma), mental health presentations, alcohol intoxication, patients deemed unable to consent due to a pre-existing medical problem (e.g. dementia) and prisoners.

Patients were screened for eligibility by the clinical team or a member of the research team. Recruitment was carried out following a 2-stage consent process. Study procedure was initiated following initial verbal ascent (by patient, personal/professional consultee) following a brief explanation. This was followed by a full informed consent.

On recruitment, we recorded demographic data, presenting complaint, past medical history, and preinclusion fluid administered (including pre-hospital). Patients were followed up to record ED diagnosis, final discharge diagnosis, length of stay and mortality data. All biochemical results were recorded.

After the initial consent process standard monitoring was used to record standard physiologic parameters throughout the patients' stay. From the standard usual care monitoring system the following parameters were recorded at baseline, and at the end of the study:

- Heart rate (HR)

- Respiratory rate

- Temperature

- Systolic blood pressure (SBP)

- Diastolic blood pressure (DBP)

- Oxygen saturation (SpO2)

The TEB monitoring electrodes were applied following manufacturers recommendations - two to each side of the neck and two to each side of the patient's lower thorax. The study procedure was carried out as follows:

- Patients were monitored for at least 3 minutes in a $45^{\circ}$ head-up position (baseline recording)

- A 3-minute PLR test was carried out by trolley manipulation for up to $45^{\circ}$ (PLR1) 
- Return to baseline position for at least 3 minutes (baseline 2)

- Fluid bolus administration (typically $500 \mathrm{~mL}$ over 15 minutes)

- PLR was repeated following at least 10 minutes from fluid bolus end (PLR2)

TEB data were transferred to Microsoft Excel (Microsoft Corporation, United States) and data on CO, SV and HR were extracted. A minute by minute average was calculated for each parameter across the whole time series. To evaluate the overall trend, each parameter was averaged for each minute across the study cohort. To estimate preload responsiveness we considered the haemodynamic variables at seven time periods:

- Baseline1: the minute immediately before leg raise

- PLR1: the middle 1-minute of PLR1

- Baseline2: the minute immediately before fluid bolus

- FB: immediately following the end of fluid bolus

- Baseline 3: 10 minutes following the end of fluid bolus

- PLR2: the middle 1-minute of the second PLR2

- Baseline4: at least 2 minutes following PLR2

Preload responsiveness was defined as $\geq 15 \%$ increase in SV. Accordingly, patients were classified into PLR+/PLR- (responders and non-responders to PLR), and R/NR (responders and non-responders to fluid bolus).

Descriptive data were presented as means with 95\% confidence interval $(\mathrm{Cl})$, medians with interquartile ranges (IQR) and proportions as appropriate. Data analysis was performed using Graphpad Prism 7 (California, United States). Correlation between the stroke volume changes ( $\Delta S V$ ) with PLR1 and FB was calculated using Pearson's r. Categorical agreement between PLR1 and FB was evaluated using Cohen Kappa. Parametric and non-parametric tests were used as appropriate to evaluate statistical significance of baseline variables between responders and non-responders.

\section{Patient and public involvement}

Patients and public were involved in the design stage through a meeting with the Leicester Cardiovascular Research Review Group. The research protocol was presented to the group and the burden of the intervention was discussed. Feedback from the group was considered during the design and conduct of the study.

\section{Results}

Thirty-nine patients met the inclusion criteria. One patient did not tolerate PLR and another had missing data due to technical failure of data transfer, so 37 patients were included in the analysis. The median age was 63 (IQR 50-77) years and 19 patients (51\%) were females. At baseline, the median early warning 
score (NEWS) was 5 (IQR 3-6) and the majority of patients had a normal blood pressure and a tachycardia. 22 patients $(60 \%)$ had an infection-related/sepsis as their ED diagnosis. Overall, patient characteristics by response to a fluid bolus are shown in Table 1. 
Table 1

Patient characteristics at baseline

\begin{tabular}{|c|c|c|c|c|}
\hline & $\begin{array}{l}\text { All } \\
N=37\end{array}$ & $\begin{array}{l}\begin{array}{l}\text { Fluid } \\
\text { Responders }\end{array} \\
\mathrm{N}=17\end{array}$ & $\begin{array}{l}\text { Fluid Non- } \\
\text { responders } \\
\mathrm{N}=20\end{array}$ & $P$ value \\
\hline Age (years) & $63(50-77)$ & $66(54-76)$ & $57(47-78)$ & ns \\
\hline Sex & $19(51 \%)$ & $9(53 \%)$ & $10(50 \%)$ & ns \\
\hline Female & $18(49 \%)$ & $8(47 \%)$ & $10(50 \%)$ & \\
\hline \multicolumn{5}{|l|}{ Male } \\
\hline Ethnicity & $31(84 \%)$ & $14(82 \%)$ & 17 (85\%) & ns \\
\hline Caucasian & $4(11 \%)$ & $2(12 \%)$ & $2(10 \%)$ & \\
\hline Asian & $2(5 \%)$ & $1(6 \%)$ & $1(5 \%)$ & \\
\hline \multicolumn{5}{|l|}{ Afro-Caribbean } \\
\hline Weight (kg) & $72(59-84)$ & $63(57-78)$ & $79(64-93)$ & ns \\
\hline Height (cm) & $174(159-180)$ & $162(152-177)$ & $175(165-180)$ & ns \\
\hline BMI & $25(21-30)$ & $25(22-30)$ & $26(21-31)$ & ns \\
\hline Heart rhythm - n (\%) & $31(84 \%)$ & $15(88 \%)$ & $16(80 \%)$ & ns \\
\hline Sinus & $5(13 \%)$ & $1(6 \%)$ & $4(20 \%)$ & \\
\hline AF & $1(3 \%)$ & $1(6 \%)$ & 0 & \\
\hline \multicolumn{5}{|l|}{ Incomplete data } \\
\hline Pre-inclusion fluid (ml) & $206 \pm 363$ & $215 \pm 339$ & $197 \pm 392$ & ns \\
\hline Study fluid & $619 \pm 224$ & $650 \pm 245$ & $593 \pm 200$ & ns \\
\hline Volume (mL) & $9 \pm 4$ & $10 \pm 4$ & $8 \pm 4$ & ns \\
\hline Volume (mL/kg) & $14 \pm 7$ & $17 \pm 9$ & $12 \pm 4$ & ns \\
\hline Infusion duration (min) & $53 \pm 24$ & $50 \pm 26$ & $56 \pm 22$ & ns \\
\hline \multicolumn{5}{|l|}{ Infusion rate (mL/min) } \\
\hline SBP $(\mathrm{mmHg})$ & $119(100-136)$ & $119(100-143)$ & $118(101-136)$ & ns \\
\hline $\mathrm{DBP}(\mathrm{mmHg})$ & $84(76-99)$ & $72(65-91)$ & $70(62-80)$ & ns \\
\hline MAP $(\mathrm{mmHg})$ & $70(64-83)$ & $88(78-109)$ & $84(75-96)$ & ns \\
\hline Heart rate (bpm) & $108(85-125)$ & $109(92-129)$ & $105(84-122)$ & ns \\
\hline Respiratory rate & $22(19-26)$ & $24(17-29)$ & $22(20-24)$ & ns \\
\hline
\end{tabular}




\begin{tabular}{|c|c|c|c|c|}
\hline & $\begin{array}{l}\text { All } \\
\mathrm{N}=37\end{array}$ & $\begin{array}{l}\begin{array}{l}\text { Fluid } \\
\text { Responders }\end{array} \\
\mathrm{N}=17\end{array}$ & $\begin{array}{l}\text { Fluid Non- } \\
\text { responders } \\
\mathrm{N}=20\end{array}$ & $P$ value \\
\hline Temperature (C) & $37(36.5-38.3)$ & $36.6(36.2-38.3)$ & $37.3(36.6-38.2)$ & ns \\
\hline $\mathrm{O}_{2}$ saturation & $97(94-100)$ & $97(95-100)$ & 97 (92-99) & ns \\
\hline New oxygen need - n (\%) & $8(22 \%)$ & $3(18 \%)$ & $5(25 \%)$ & ns \\
\hline NEWS & $5(3-6)$ & $5(3-7)$ & $4(3-6)$ & ns \\
\hline Base excess & $0.9(-4.3-3.75)$ & $0.55(-5.53-2.58)$ & $1.2(-3.3-4.5)$ & ns \\
\hline Lactate & $\begin{array}{l}1.9(1.25- \\
3.65)\end{array}$ & $1.75(1.15-4.28)$ & $2.0(1.3-3.6)$ & ns \\
\hline Stroke volume (mL) & $60(49-82)$ & $51(47-56)$ & $72(60-90)$ & 0.0066 \\
\hline Cardiac output (L/min) & $6.1(4.4-8.1)$ & $5.0(4.1-6.3)$ & $7.4(5.7-9.1)$ & 0.0044 \\
\hline Impedance & $34(31-38)$ & $34(31-38)$ & $33(29-38)$ & ns \\
\hline $\begin{array}{l}\text { Signal quality indicator } \\
(\%)\end{array}$ & $47(29-68)$ & $57(32-70)$ & $44(27-66)$ & ns \\
\hline
\end{tabular}

All patients received a crystalloid fluid bolus with the majority of patients receiving $500 \mathrm{~mL}$ with a median of $7.9 \mathrm{~mL} / \mathrm{kg}$ (actual body weight), IQR 6.1-11. The median time of fluid bolus infusion was 12 minutes (IQR 9-15.5), with a median fluid infusion rate of $53 \mathrm{~mL} / \mathrm{min}$ (IQR 33-73).

Thirty-five patients were admitted to a hospital ward (level 0), one patient to intensive care unit (level 3) and another patient to enhanced acute ward (level 1). Patients had a median hospital stay of 5.5 days (IQR 2.25-8), with no significant difference between those who responded to the initial fluid bolus and those who did not ( 6 vs 5 days, $p=0.252$ ). One patient died in hospital.

\section{Overall haemodynamic changes}

TEB monitoring showed that the patients had a median baseline SV of $60 \mathrm{~mL}$ which increased to a median of $71 \mathrm{~mL}$ ( $18 \%$ increase) immediately following fluid infusion and then fell back to a median of $67 \mathrm{~mL}$ at 10 minutes and was $63 \mathrm{~mL}$ (near baseline) by 20 minutes. The baseline PLR1 Test gave a median increase in SV of 10\%, with the PLR2 test (after fluid infusion) giving the same result (Fig. 1). The median $\mathrm{CO}$ showed a similar pattern, albeit with less pronounced changes during the PLR tests. Heart rate data did not show any observable change with PLR or fluid bolus. 


\section{Haemodynamic changes with baseline PLR}

Eleven patients (30\%) showed a positive SV response $(\geq 15 \%)$ to the baseline PLR test. with a median SV increase of $30 \%$ following the PLR manoeuvre, which then rapidly fell back to baseline. In these patients, the median SV increased following fluid bolus by $22 \%$ with partial resolution at 10 minutes after infusion. When the PLR test was repeated after fluid infusion in this patient group there was a median increase of SV by $18 \%$ again rapidly reversed after the manoeuvre.

In the patients with a negative baseline PLR test there was no change in median SV with the fluid bolus and again no change in SV with the PLR test after fluid infusion. (Fig. 2).

\section{Haemodynamic changes with fluid bolus}

\section{- Fluid responders:}

Seventeen patients (46\%) responded to the fluid bolus ( $\geq 15 \%$ SV change). In these 'fluid responders' the median SV increase following fluid bolus was $28 \%$, with partial resolution to $22 \%, 10$ minutes later. This group of patients had a median SV increase of $16 \%$ during the baseline PLR test, with a rapid fall in SV back to the baseline after the test. When the PLR test was repeated after the fluid bolus there was a median SV increase of $10 \%$, which again rapidly reversed after the test.

\section{- Fluid non-responders:}

In the patients who did not have a change in SV (median - 9\% in response to the fluid bolus, there was only a small 3\% rise in SV during the baseline PLR test, with a larger (but still borderline for a 'response') increase in SV (15\%)in response to the PLR test performed after the fluid bolus (Fig. 2).

\section{Relationship between PLR1 and FB haemodynamic changes}

Change in SV $(\Delta S V)$ during the baseline PLR1 test showed a moderate correlation with $\triangle S V$ after fluid bolus - Pearson r of 0.47 (95\% $\mathrm{Cl} 0.17-0.69)$ and $62 \%$ concordance (23 patients), Fig. 3. It is noticeable that all of the patients who had a large adverse effect (decrease in SV) from the fluid bolus (those to the left of the ' $y$ ' axis) were "non-responders" to the baseline PLR test (are below the 15\% horizontal dotted threshold for fluid responsiveness). Conversely, there were a number of patients who had a large increase in SV following the fluid bolus (those to the right of the ' $y$ ' axis), but were "non-responders" to the Baseline PLR test (below the dotted FR threshold).

To predict fluid responsiveness ( $\triangle S V \geq 15 \%$ with fluid bolus), the Baseline PLR test had a sensitivity of $41 \%(95 \% \mathrm{Cl} 22-64)$ and specificity of $80 \%(95 \% \mathrm{Cl} 58-92)$. This gave a positive predictive value of $64 \%$ (95\% $\mathrm{Cl} 35-85)$ and a negative predictive value of $62 \%(95 \% \mathrm{Cl} 43-78)$. The positive likelihood ratio was 
2.06 (95\% $\mathrm{Cl} 0.72-5.85)$, while the negative likelihood ratio was $0.74(95 \% \mathrm{Cl} 0.47-1.16)$. PLR1 had an area under the curve of $0.59(95 \% \mathrm{Cl} 0.41-0.78)-\mathrm{P}$ value 0.33 .

We analysed the degree of concordance between PLR1 and FB using different thresholds $(0-15 \%)$ to define 'positive response' to both the baseline PLR test and the fluid bolus. The highest concordance (62\%) was achieved using the $15 \%$ threshold for both PLR1 and FB (Table 2).

Table 2

Concordance between Baseline PLR test and fluid bolus using different positive response thresholds

\begin{tabular}{|lllll|}
\hline & $>0 \%$ FB & $\geq 5 \%$ FB & $\geq 10 \%$ FB & $\geq 15 \%$ FB \\
\hline$>0 \%$ Baseline PLR & $60 \%$ & $57 \%$ & $46 \%$ & $49 \%$ \\
\hline$\geq 5 \%$ Baseline PLR & $60 \%$ & $57 \%$ & $46 \%$ & $49 \%$ \\
\hline$\geq 10 \%$ Baseline PLR & $60 \%$ & $60 \%$ & $54 \%$ & $58 \%$ \\
\hline$\geq 15 \%$ Baseline PLR & $46 \%$ & $54 \%$ & $58 \%$ & $62 \%$ \\
\hline
\end{tabular}

\section{Accuracy of baseline parameters to predict fluid responsiveness}

The parameters conventionally used in emergency care to assess volume status and determine fluid management, such as pulse rate and blood pressure, showed poor performance in predicting fluid responsiveness (Fig. 4). The AUC for these parameters are shown in Fig. 4, showing lower predictive value than baseline SV and CO - which seemed to be better predictors of response (Fig. 4).

\section{Discussion}

In this work, we found that SV changes with PLR test were moderately correlated with SV changes following a fluid bolus. In a sample of 37 patients, the test showed a reasonable specificity of $80 \%$, but a poor sensitivity of $41 \%$. This suggests that a patient with a positive Baseline PLR test is likely to respond to a fluid bolus, but that a patient with a negative baseline test may or may not respond.

The majority of our patient cohort were elderly and not critically unwell - which is typical of a UK ED sepsis population. In the UK, sepsis protocols use EWS along with suspected infection to trigger a fluid challenge as part of the 'sepsis six' bundle, so it is normal for this treatment to be given to patients before they become critically unwell.

Conceptually, preload challenge is assumed to increase venous return, then increasing right ventricular diastolic volume, with a subsequent increase in left ventricular SV (preload responsiveness). Fluid 
challenge is the gold standard for assessing preload responsiveness, however variation in the challenge technique (volume and rate) may affect the outcome. A meta-analysis by Toscani et al. showed heterogenicity in technique and that an infusion time $>30$ minutes was associated with a lower proportion of responsiveness. ${ }^{10} \mathrm{~A}$ systematic review of ICU and ED fluid challenges found that the infusion time was variable (median of 30 minutes, range 3 to 60 ). ${ }^{11}$ Similar large variation in practice was seen in the FENICE study, with a median of 24 minutes. ${ }^{12}$ The effect of variation in the dose of fluid challenge on fluid responsiveness was examined by Aya et al. with a conclusion that a dose of at least $4 \mathrm{~mL} / \mathrm{kg}$ should be used. ${ }^{13}$

Stroke volume change ( $\Delta S V$ ) in both PLR tests (before and after fluid bolus) was positive in $30 \%$ of patients, which is within the range reported in previous literature. ${ }^{9}$ However a higher proportion of patients were responsiveness ( $\triangle S V$ ) to the fluid bolus (46\%). This higher preload response to FB compared to PLR has previously been seen in both mechanically ventilated and spontaneously breathing patients. ${ }^{14} 15$ There are several possible explanations for this observation. The fluid bolus may give a greater preload challenge than PLR (which only cause around $300 \mathrm{~mL}$ of fluid shift - which may be even less in the dehydrated patient). Undertaking PLR in awake patients may also be associated with neural responses (e.g. vagal stimulation) which may influence the $\Delta S V^{16}$

Stroke volume changes $(\Delta S V)$ with baseline PLR showed a moderate correlation with $\Delta S V$ following fluid bolus with $62 \%$ positive concordance (Fig. 4). Past research on fluid challenge has concentrated on identifying the patients who need fluid resuscitation, however it can be seen that all patients who had a large negative response to $\mathrm{FB}(>15 \%$ decrease in SV) were predicted by a negative Baseline PLR test PLR. Future studies might use the PLR test to identify those patients who might be adversely affected by a fluid bolus.

While PLR had limited diagnostic performance in predicting FR, none of the standard monitoring parameters showed better performance. Notably, Baseline SV and CO showed a higher predictive ability than any of the currently used parameters (such as pulse rate and blood pressure), which is consistent with our volunteer results. ${ }^{16}$ This generates an interesting hypothesis of whether baseline SV (without the PLR) could add value to the assessment of volume status in ED.

In our experiment, PLR was able to stratify patients into two different groups according to fluid responsiveness. However, it seemed to be testing a cardiovascular response that was related to, but different from the response to a fluid bolus. Using a diagnostic threshold of an increase in SV $>15 \%$ failed to show adequate accuracy for PLR alone as a definitive diagnostic test to predict fluid responsiveness, however as it performed better than any of the currently used parameters.

\section{Limitations}

Our study included a convenience sample of ED patients and may be liable for selection bias. A single operator (ME) carried out the procedure so we cannot present any data on inter-rater reliability. Operator 
was not blinded to SV changes during PLR and fluid bolus. However, this is less likely to have introduced bias as the analysis was carried out using a standardised code blinded to individual SV changes.

In our study, data analysis did not happen in real-time and was based on summary numbers over time windows. This is not strictly comparable to the clinical situation where the clinician at the bedside is able to look at the continuous SV trace. This gives additional information about the reliability of the data, such as the stability of signal and the degree of artefacts observed (similar to the interpretation of pulse oximetry at the bedside). This additional information (which could not be used in this study without introducing observer bias) might change the utility of SV monitoring in real life.

A potential limitation of the study is that, as expected in older people, five patients had atrial fibrillation. The TEB monitoring technique is known to be less accurate in these patients, however they were included in the study to ensure that the results were generalisable to a normal ED sepsis population. A further limitation is that compared with previous ICU studies the ED patients had relatively little fluid before the PLR test - this may have reduced the fluid shift caused by the PLR, and given a false negative result. However, most ED patients have little fluid resuscitation on presentation, so the study population is representative of normal practice.

Another caveat is the specific time windows for evaluating a dynamic process (e.g. evaluating fluid responsiveness in a fixed time window immediately following fluid infusion). While this method has some evidence base, there is a disadvantage in artificially imposing time windows. ${ }^{17}$ In real life a clinician would continuously inspect the pattern of change over time (rather than forcing a judgment within a specific time) - a complex process of clinical judgement which is difficult to replicate mathematically. Another potential limitation is forcing patients into binary responder/non-responder groups using the artificial $15 \%$ change. There are probably degrees of response, for example our data suggest that negative responder $(>-15 \% \Delta S \mathrm{~S})$, positive responder ( $>+15 \% \Delta S \mathrm{~S})$ and intermediate response may be useful future categories.

\section{Conclusion}

The PLR test showed a moderate correlation with SV changes following a subsequent fluid bolus, with a limited accuracy to predict fluid responsiveness. A group of patients who have an adverse response to the conventional sepsis management protocol might be predicted by a negative SV change on baseline PLR test. The PLR test was a better predictor of fluid responsiveness than the parameters commonly used in emergency care (such as heart rate and blood pressure) which had poor accuracy. In the context of what is already known about cardiac monitoring and fluid challenges, these data suggest the potential for a clinical trial in sepsis comparing TEB monitored, PLR directed fluid management with standard care.

\section{Declarations}

\section{Ethics approval and consent to participate}


The study was sponsored by the University of Leicester, UK and ethical approvals were obtained from the Essex Research Ethics Committee (16/EE/0145). A full informed consent was obtained from all participants.

\section{Consent for publication}

Not applicable.

\section{Availability of data and material}

Please contact author for data requests.

\section{Competing Interests}

The authors declare that they have no competing interests.

\section{Funding}

ME is funded by Newton-Mosharafa PhD Fund. ME and TC secured further project funding through the RCEM grant award scheme (no. G/2017/1).

\section{Authors' contributions}

ME conducted the study procedure, data analysis and final write-up. AR contributed to study design, critical revision and editing. EE and SE contributed to study design and supervision. TC contributed to study design, critical revision and editing. All the authors reviewed and approved the final manuscript. ME is responsible for the overall content as guarantor.

\section{Acknowledgements}

ME would like to thank the Ministry of Higher Education - Missions Sector, Egypt and the British Council for their support through Newton-Mosharafa Fund.

\section{References}


1. Investigators TP. Early, Goal-Directed Therapy for Septic Shock - A Patient-Level Meta-Analysis. $N$ Engl J Med 2017 doi: 10.1056/NEJMoa1701380 [published Online First: 2017/03/23].

2. Rhodes A, Evans LE, Alhazzani W, et al. Surviving Sepsis Campaign: International Guidelines for Management of Sepsis and Septic Shock: 2016. Crit Care Med. 2017;45(3):486-552. doi: $10.1097 / \mathrm{ccm} .0000000000002255$.

3. Lakhal K, Ehrmann S, Perrotin D, et al. Fluid challenge: tracking changes in cardiac output with blood pressure monitoring (invasive or non-invasive). Intensive Care Med. 2013;39(11):1953-62. doi:10.1007/s00134-013-3086-6 [published Online First: 2013/09/26].

4. Harris T, Coats TJ, Elwan MH. Fluid therapy in the emergency department: an expert practice review. Emerg Med J. 2018;35(8):511. doi:10.1136/emermed-2017-207245.

5. Powell MF, Mathru M, Brandon A, et al. Assessment of endothelial glycocalyx disruption in term parturients receiving a fluid bolus before spinal anesthesia: a prospective observational study. Int $\mathrm{J}$ Obstet Anesth. 2014;23(4):330-4. doi:10.1016/j.jjoa.2014.06.001 [published Online First: 2014/09/10].

6. Monnet $X$, Teboul J. Passive leg raising for predicting fluid responsiveness: a systematic review and meta-analysis. Intensive Care Medicine Experimental. 2015;3(Suppl 1):A587.

7. Monnet X, Teboul J-L. Passive leg raising: five rules, not a drop of fluid! Crit Care. 2015;19(1):18.

8. Elwan MH, Hue J, Green SJ, et al. Thoracic electrical bioimpedance versus suprasternal Doppler in emergency care. Emerg Med Australas. 2017;29(4):391-93. doi:10.1111/1742-6723.12765.

9. Elwan MH, Roshdy A, Elsharkawy EM, et al. The haemodynamic dilemma in emergency care: Is fluid responsiveness the answer? A systematic review. Scand J Trauma Resusc Emerg Med. 2017;25(1):25. doi:10.1186/s13049-017-0370-4.

10. Toscani $L$, Aya HD, Antonakaki $D$, et al. What is the impact of the fluid challenge technique on diagnosis of fluid responsiveness? A systematic review and meta-analysis. Crit Care. 2017;21:207. doi:10.1186/s13054-017-1796-9.

11. Glassford NJ, Eastwood GM, Bellomo R. Physiological changes after fluid bolus therapy in sepsis: a systematic review of contemporary data. Crit Care. 2014;18(6):1.

12. Cecconi M, Hofer $C$, Teboul J-L, et al. Fluid challenges in intensive care: the FENICE study. Intensive Care Med. 2015;41(9):1529-37.

13. Aya HD, Rhodes A, Chis Ster I, et al. Hemodynamic Effect of Different Doses of Fluids for a Fluid Challenge: A Quasi-Randomized Controlled Study. Crit Care Med. 2017;45(2):e161-e68. doi: $10.1097 / \mathrm{ccm} .0000000000002067$.

14. Miller J, Ho C-X, Tang J, et al. Assessing Fluid Responsiveness in Spontaneously Breathing Patients. Acad Emerg Med. 2016;23(2):186-90. doi:10.1111/acem.12864.

15. Benomar B, Ouattara A, Estagnasie P, et al. Fluid responsiveness predicted by noninvasive Bioreactance-based passive leg raise test. Intensive Care Med. 2010;36(11):1875-81. doi:10.1007/s00134-010-1990-6. 
16. Elwan MH, Roshdy A, Reynolds JA, et al. What is the normal haemodynamic response to passive leg raise? A study of healthy volunteers. Emerg Med J. 2018. doi:10.1136/emermed-2017-206836.

17. Aya HD, Ster IC, Fletcher N, et al. Pharmacodynamic analysis of a fluid challenge. Crit Care Med. 2016;44(5):880-91.

\section{Figures}
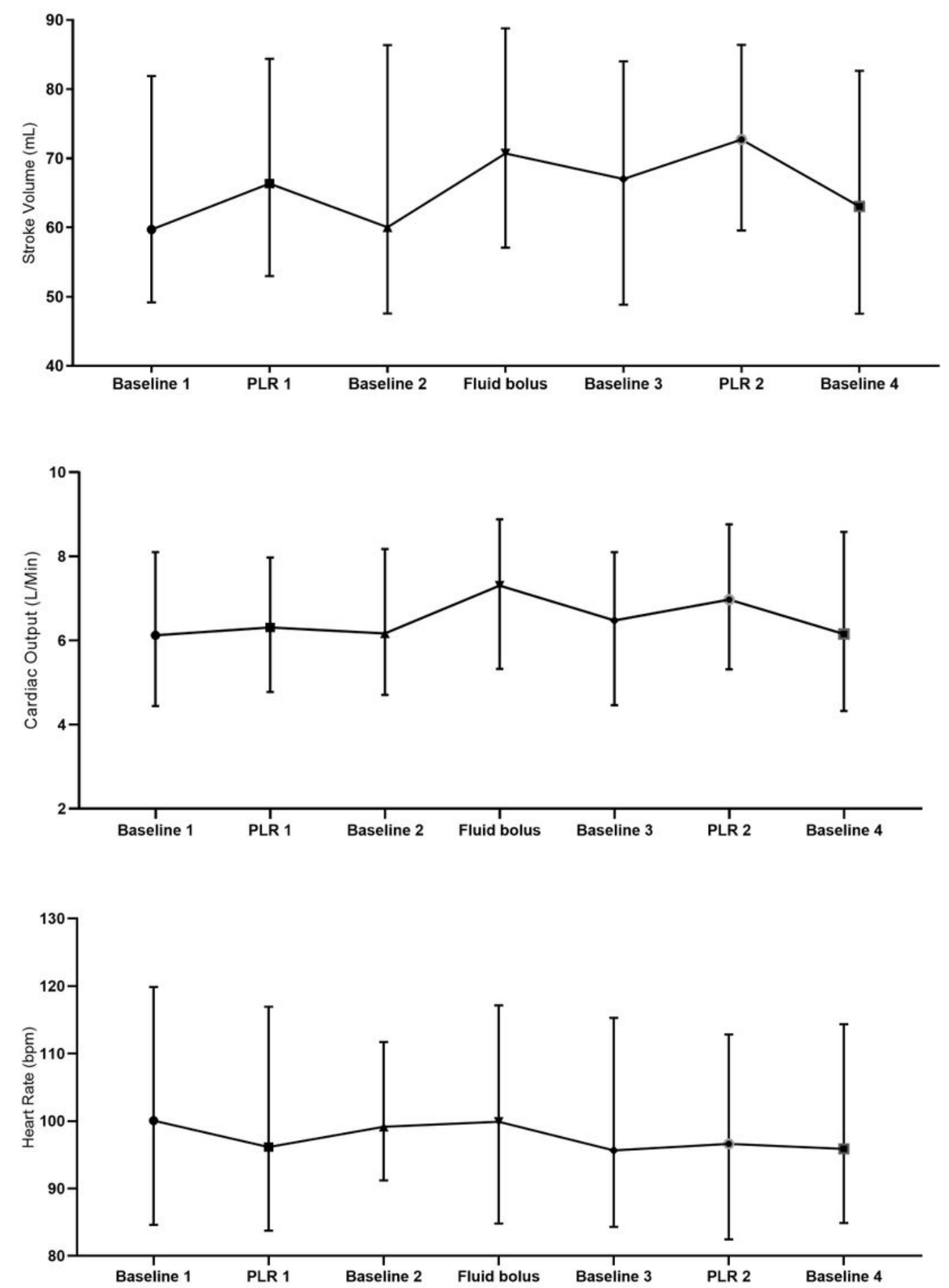
Figure 1

Overall haemodynamic changes. PLR, passive leg raise
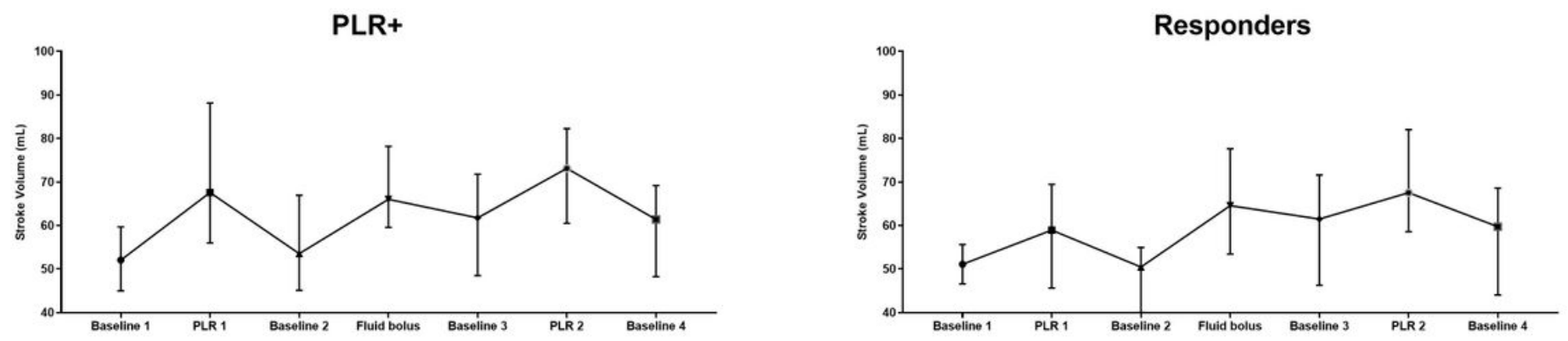

PLR-
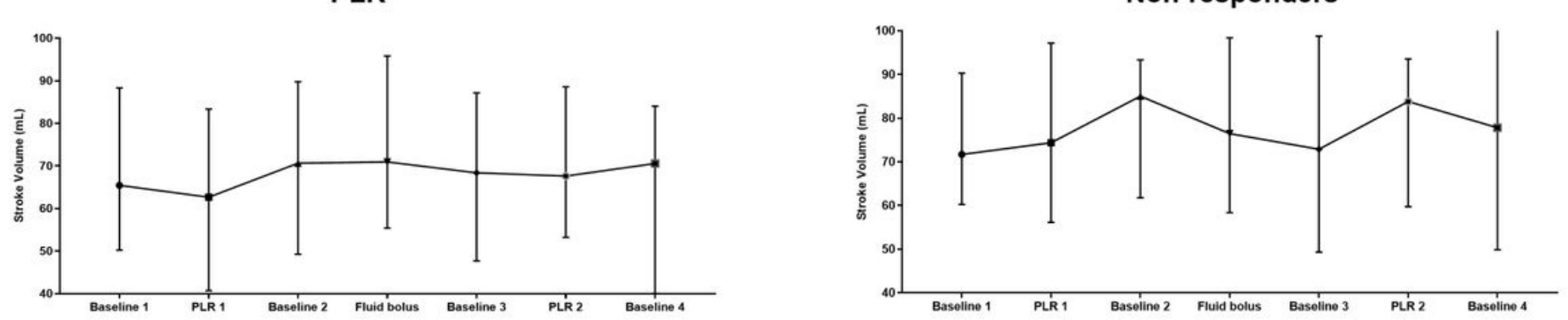

Figure 2

Stroke volume changes in classified by responsiveness to passive leg raise (PLR +ve/-ve) and by fluid responsiveness (responders/non-responders). 


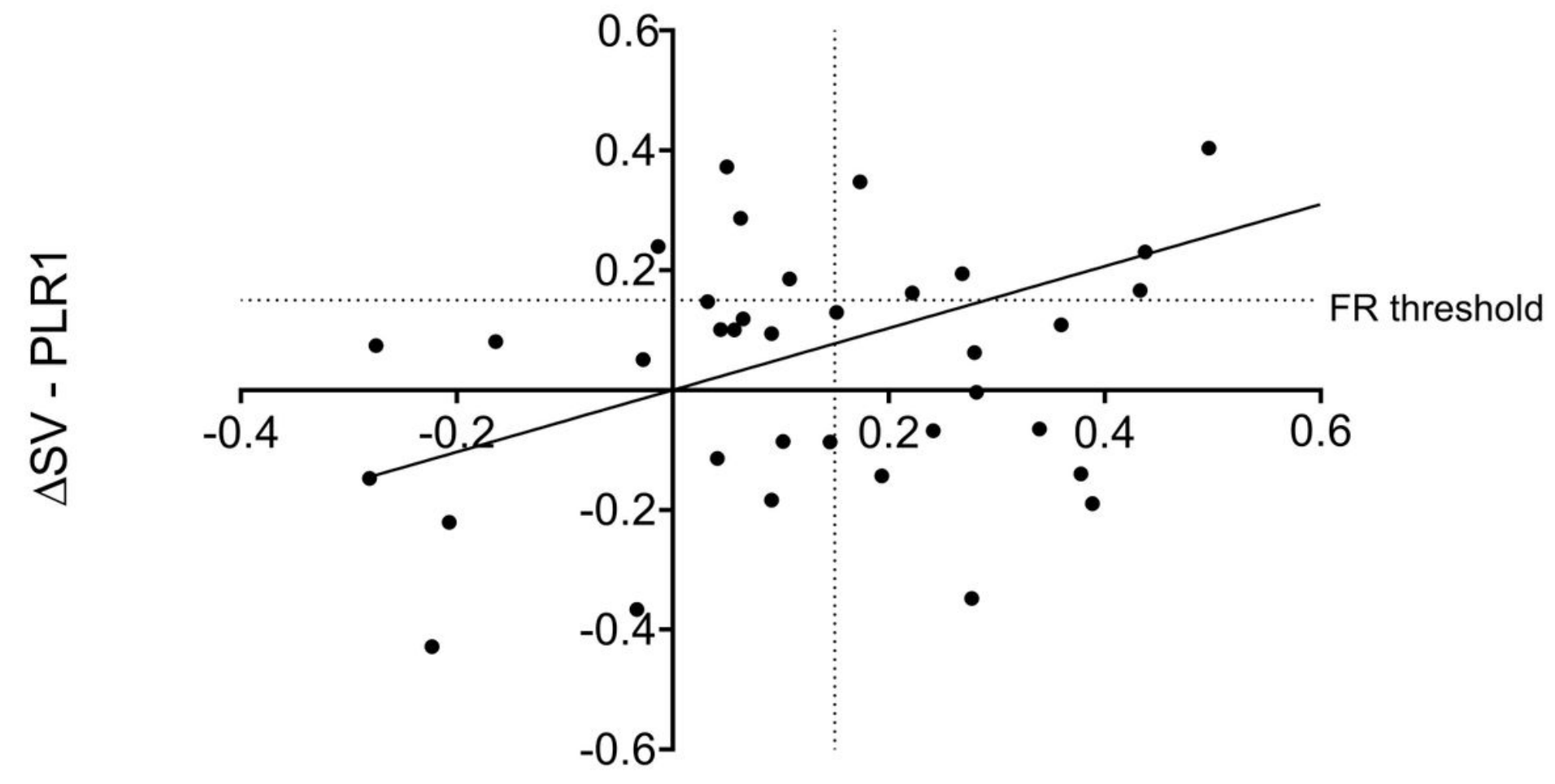

$\Delta S V$ - fluid bolus

Figure 3

Correlation between stroke volume changes (SV) with fluid bolus and passive leg raise 1 (PLR1). One point is outside the axis limit. FR, fluid responsiveness. 

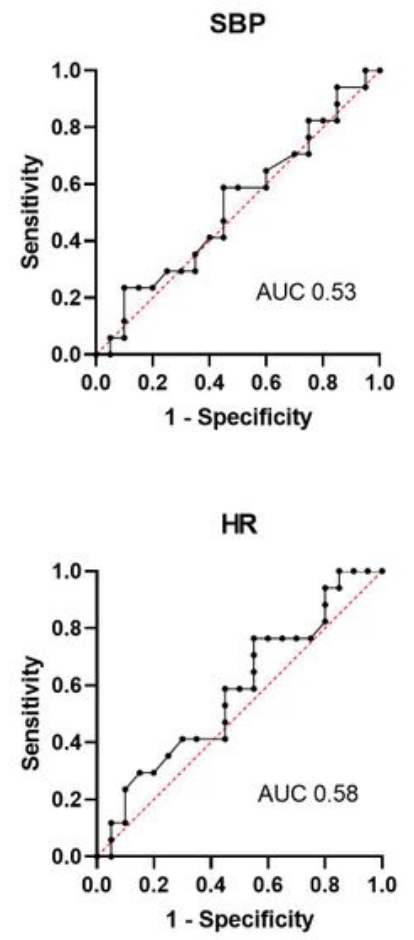

Lactate

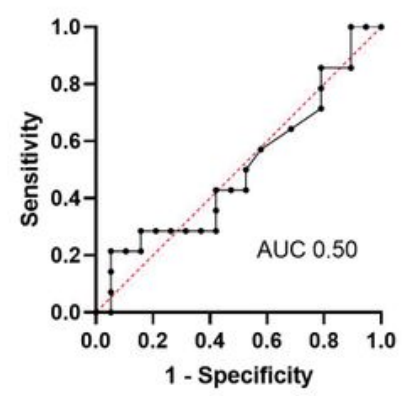

Baseline SV

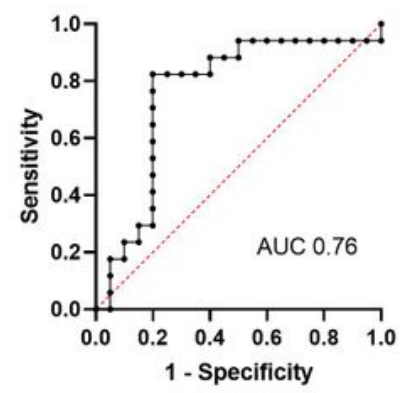

MAP

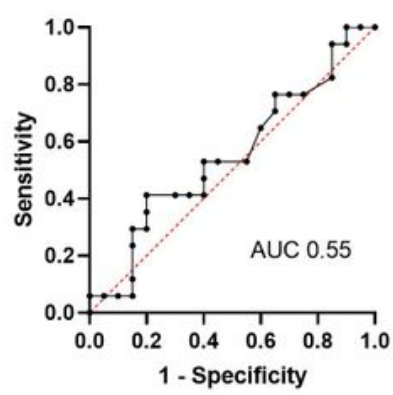

EWS

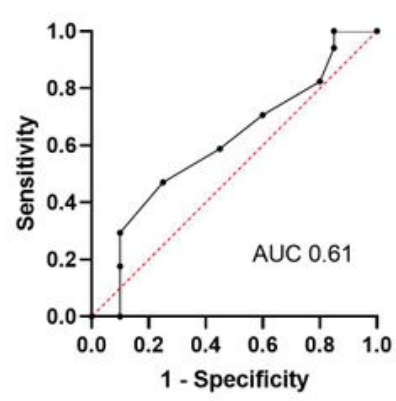

Base excess

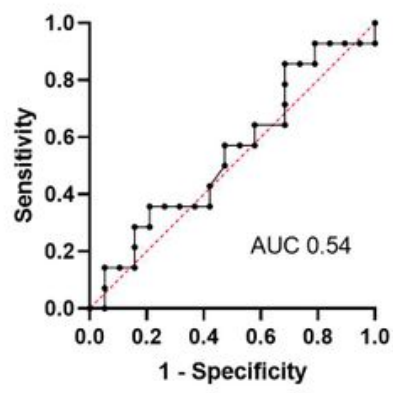

Baseline CO

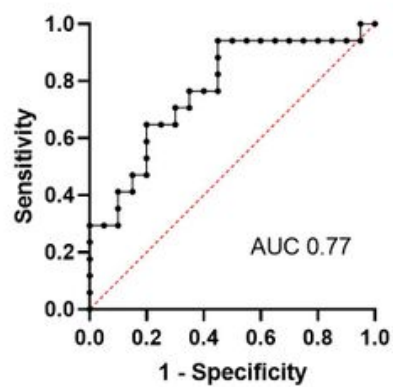

\section{Figure 4}

Receiver operator curve for the accuracy of baseline parameters in predicting fluid responsiveness. CO, cardiac output; EWS, early warning score; HR, heart rate; MAP, mean arterial pressure; SV, stroke volume; SBP, systolic blood pressure.

\section{Supplementary Files}


This is a list of supplementary files associated with this preprint. Click to download.

- STARD2015Checklist.docx 